\title{
Impaired immune response to Candida albicans in aged mice
}

\section{Correspondence \\ M. Luisa Gil \\ m.luisa.gil@uv.es}

Received 25 May 2006

Accepted 14 August 2006

\section{Celia Murciano, ${ }^{1}$ Eva Villamón, ${ }^{1}$ Alberto Yáñez, ${ }^{1}$ José-Enrique O'Connor, ${ }^{2}$ Daniel Gozalbo ${ }^{1}$ and M. Luisa Gil ${ }^{1}$}

\author{
${ }^{1}$ Departamento de Microbiología y Ecología, Universitat de València, Facultad de Ciencias \\ Biológicas, Edificio de Investigación, C/Dr. Moliner 50, 46100 Burjasot, Valencia, Spain \\ ²Laboratorio de Citómica, Unidad Mixta CIPF-UVEG, Centro de Investigación 'Principe Felipe', \\ Valencia, Spain
}

\begin{abstract}
The prevalence of opportunistic fungal infections has increased dramatically among the aged population in recent years. This work investigated the effect of ageing on murine defences against Candida albicans. Aged C57BL/6 mice that were experimentally infected intravenously had a significantly impaired survival and a higher tissue fungal burden compared with young mice. In vitro production of tumour necrosis factor (TNF)- $\alpha$ by macrophages from aged mice in response to yeast cells and hyphae of $C$. albicans was significantly lower than production by macrophages from young mice. In vitro production of cytokines, such as TNF- $\alpha$ and gamma interferon (IFN- $\gamma$ ), by antigen-stimulated splenocytes from mice intravenously infected with $C$. albicans cells was also diminished in old mice. This decrease in production of T helper 1 cytokines in old mice correlated with a diminished frequency of IFN- $\gamma$-producing $\mathrm{CD} 4^{+} \mathrm{T}$ lymphocytes, although the ability to develop an acquired resistance upon vaccination (primary sublethal infection) of mice with the low-virulence PCA2 strain was not affected in aged mice. The diversity of antigens recognized by C. albicans-specific antibodies in sera from infected aged mice was clearly diminished when compared with that from infected young mice. Taken together, these data show that aged mice develop an altered innate and adaptive immune response to C. albicans and are more susceptible to systemic primary candidiasis.
\end{abstract}

\section{INTRODUCTION}

In humans, Candida albicans is the micro-organism most frequently associated with systemic fungal infections in immunocompromised patients. The delicate balance between the host and this commensal non-professional pathogen turns into a parasitic relationship resulting in the development of invasive infections. The nature and extent of the impairment of the host defence influence the manifestation and severity of infection. The incidence of these infections is increasing as a result of a growing population of immunocompromised individuals, due to the use of intensive chemotherapy and immunosuppressive drugs. Systemic candidiasis can be life-threatening and is associated with high morbidity and mortality, as diagnosis is difficult and current antifungal therapies often fail (Calderone, 2001; Garber, 2001).

Resistance to candidiasis requires the coordinated action of innate and adaptive immune defences. Neutrophils and

Abbreviations: IFN- $\gamma$, gamma interferon; IL, interleukin; i.v., intravenous(ly); Th1, T helper 1; TLR, Toll-like receptor; TNF, tumour necrosis factor. macrophages can clear the pathogen via phagocytosis, and macrophage activation also leads to the release of several key mediators such as pro-inflammatory cytokines, which are important for protecting the host against disseminated candidiasis. It is accepted that antifungal $\mathrm{CD} 4{ }^{+} \mathrm{T}$ helper 1 (Th1)-mediated responses play a central role in anti- $C$. albicans defences, providing control of fungal infectivity through the production of gamma interferon (IFN- $\gamma$ ), which is required for optimal activation of phagocytes and for helping in the generation of a protective antibody response. A special feature of C. albicans is the morphological transition from the yeast to the hyphal form, which is strictly associated with virulence, as several agerminative mutants invariably have low systemic pathogenicity. Phagocytosis of the yeast form induces murine dendritic cells to produce interleukin (IL)-12 and to prime Th1 lymphocytes, whereas ingestion of the hyphal form results in IL-4 production, which favours Th2 cell priming (Calderone, 2001; Romani, 2004).

In the elderly, many alterations of both innate and adaptive immunity have been described, generally viewed as a deterioration of immunity, leading to the use of the term immunosenescence (Effros, 2001; Ginaldi et al., 2001; 
McGlauchlen \& Vogel, 2003; Plackett et al., 2004). Most research concerning immunosenescence has been carried out on $\mathrm{T}$ and $\mathrm{B}$ cells, which show an altered cytokine pattern, a reduction in clonal expansion and function of antigenspecific $T$ and $B$ cells, and a decline in antigen-presentingcell function (Effros, 2001; McGlauchlen \& Vogel, 2003). Similarly, the functions of macrophages, neutrophils and natural killer cells, components of the innate immunity, are also decreased (Plackett et al., 2004). The decline in immune function leads to increased susceptibility of aged individuals to viral, bacterial and fungal infections, reactivation of latent viruses and a decreased response to vaccines (Effros, 2001; Ginaldi et al., 2001). Invasive infections with opportunistic fungi, such as Candida species, have become an increasing problem in old adults because they are more likely to be considered for transplantation, to receive aggressive regimens of chemotherapy for cancer and to take immunosuppressive drugs for non-malignant diseases (Kauffman, 2001). In addition, the immunosenescence process in older adults probably enhances the risk and severity of candidaemia (Nucci et al., 1998).

In the present study, using young and aged mice, we studied the influence of ageing on (i) susceptibility in vivo to experimental candidiasis, (ii) in vitro tumour necrosis factor (TNF)- $\alpha$ release by macrophages in response to C. albicans, (iii) the production of Th1 cytokines (IFN- $\gamma$ and TNF- $\alpha$ ) by splenocytes, (iv) the development of a Th1 response, (v) the ability to develop an acquired resistance upon vaccination (primary sublethal infection) and (vi) the development of an acquired humoral response.

\section{METHODS}

Mice and yeast strains. Young (2-3 months) and aged (18-20 months) C57BL/6 female mice were purchased from Harlan Ibérica. All assays involving mice were approved by the Institutional Animal Care and Use Committee.

Endotoxin-free starved and heat-inactivated C. albicans ATCC 26555 yeast cells or hyphae and endotoxin-free starved yeast cells of $C$. albicans PCA2, a low-virulence non-germinative strain, were prepared as described previously (Villamón et al., 2004a, b, c). All of the assays were performed under conditions designed to minimize endotoxin contamination. Endotoxin-free water and PBS were used, and fungal culture media were passed through a Detoxi-Gel endotoxin-removing gel (Pierce) and tested for the absence of endotoxin by the E-Toxate assay (Sigma).

Infection model and survival curves. Young and aged mice were intravenously infected with starved yeast cells of the high-virulence C. albicans strain ATCC 26555 . Mice were challenged with $8 \times 10^{5}$ yeast cells in a volume of $0.2 \mathrm{ml}$ PBS and survival was checked daily for 21 days.

In vaccination assays, aged mice were infected with the low-virulence C. albicans strain PCA2 by intravenous (i.v.) injection of $10^{6}$ yeast cells in a volume of $0.2 \mathrm{ml}$ (primary candidiasis). Fifteen days later, immunized mice were i.v. infected (secondary infection) with $C$. albicans ATCC $26555\left(10^{6}\right.$ yeast cells in a volume of $\left.0 \cdot 2 \mathrm{ml}\right)$ and survival was checked daily for 14 days. Control aged mice were intravenously infected with $10^{6}$ yeast cells of C. albicans ATCC 26555 (primary candidiasis) and survival was checked for 14 days.

To assess the tissue outgrowth of the micro-organism, young and aged mice were i.v. infected with $8 \times 10^{5}$ C. albicans ATCC 26555 yeast cells. At 3 and 5 days post-infection (p.i.), mice were killed and the kidneys were removed aseptically, weighed and homogenized in $1 \mathrm{ml} \mathrm{PBS}$; dilutions of the homogenates were plated on Sabouraud/glucose agar. The c.f.u. were counted after $24 \mathrm{~h}$ of incubation at $37^{\circ} \mathrm{C}$ and expressed as c.f.u. (g tissue) ${ }^{-1}$. The amount of TNF- $\alpha$ in the supernatants of kidney homogenates was determined using a commercial ELISA kit (eBioscience). Mice in a moribund condition were sacrificed irrespective of the planned schedule and were not evaluated for c.f.u. or TNF- $\alpha$.

Isolation of mouse peritoneal macrophages and splenocytes. Resident peritoneal macrophages were harvested by injecting and withdrawing $10 \mathrm{ml}$ complete cell-culture medium (RPMI 1640 supplemented with $5 \%$ heat-inactivated fetal bovine serum and $1 \%$ penicillin/streptomycin; Gibco). Cells were washed once with the same medium and plated at a density of $2 \cdot 3 \times 10^{5}$ cells in $200 \mu \mathrm{l}$ medium per well in a 96-well tissue culture plate. Peritoneal macrophages were allowed to adhere for $2 \mathrm{~h}$ at $37^{\circ} \mathrm{C}$ in a $5 \% \mathrm{CO}_{2}$ atmosphere, the non-adherent cells were removed and the adherent macrophages were cultured for $72 \mathrm{~h}$ prior to challenge, as described previously (Villamón et al., 2004b).

Splenocytes were obtained as described elsewhere (Villamón et al., $2004 a, c)$. Mice were i.v. infected $\left(4 \times 10^{5}\right.$ yeast cells per mouse) with either C. albicans PCA2 or ATCC 26555 yeast cells. Three days after infection, total spleen cells were obtained by collagenase D treatment of the spleens, washed once with complete cell-culture medium and plated at a density of $10^{7}$ cells in $1 \mathrm{ml}$ medium per well in a 24-well tissue culture plate, in the presence of $2 \cdot 5 \mu \mathrm{g}$ amphotericin B (Gibco) $\mathrm{ml}^{-1}$.

Measurement of in vitro cytokine production. Cells (peritoneal macrophages or splenocytes) were challenged with the indicated stimuli for 24 or $48 \mathrm{~h}$, respectively, at $37^{\circ} \mathrm{C}$. Samples with no stimuli were used as controls. Supernatants were harvested and tested using commercial ELISA kits for TNF- $\alpha$ and IFN- $\gamma$ (eBioscience).

The stimuli used were LPS from Escherichia coli O111: B4 (Sigma), the yeast cell-wall particle zymosan (Molecular Probes) and heatinactivated C. albicans ATCC 26555 yeast cells or hyphae, obtained as reported elsewhere (Gil-Navarro et al., 1997; Gozalbo et al., 1998). Briefly, starved yeast cells were inoculated [200 $\mu \mathrm{g}$ cells (dry wt) $\mathrm{ml}^{-1}$ ] in a minimal synthetic medium and incubated for $3 \mathrm{~h}$ at $28^{\circ} \mathrm{C}$ to obtain yeast cells or at $37^{\circ} \mathrm{C}$ to obtain hyphae. For heat inactivation, yeast cells and hyphae were resuspended in PBS and treated at $100{ }^{\circ} \mathrm{C}$ for $1 \mathrm{~h}$. After inactivation, fungal cells were washed in PBS and brought to the desired cell density in complete cell-culture medium.

IFN- $\boldsymbol{\gamma}$-secretion assay. After i.v. infection of mice with $4 \times 10^{5}$ yeast cells of either C. albicans PCA2 or ATCC 26555, total spleen cells were obtained as described above, challenged in vitro with heatkilled C. albicans ATCC 26555 yeast cells or hyphae for $18 \mathrm{~h}$, and analysed for IFN- $\gamma$ secretion using the mouse IFN- $\gamma$-secretion assay detection kit (Miltenyi Biotec) according to the manufacturer's instructions. Cells were also incubated with a saturating amount of FITC-conjugated CD4 mAb (RM4-5; Pharmingen) and phycoerythrin (PE)-Cy5-conjugated CD3 mAb (145-2C11; Pharmingen). After washing twice with PBS, the stained cells were analysed on an EPICS XL-MCL flow cytometer (Beckman Coulter).

Western immunoblot. Soluble cell extracts, obtained from yeast cells or hyphae by boiling cells in PBS supplemented with $1 \%$ SDS and $1 \%$ DTT, were separated by SDS-PAGE and transferred to 
PVDF membranes (Amersham Pharmacia Biotech). Blots were incubated with a $1: 10$ dilution of mouse serum obtained before primary infection with strain PCA2 (uninfected sera) and 14 days after secondary infection with strain ATCC 26555 (see above). Reactive bands were developed using goat anti-mouse IgG horseradish peroxidase-conjugated antibody (1:1000 dilution; Sigma), and hydrogen peroxide and 4-chloro-1-naphthol as the chromogenic reagent.

Statistical analysis. Survival curves were analysed using the Kaplan-Meyer log rank test. Student's two-tailed $t$ test was used to compare cytokine production and numbers of c.f.u. Data were expressed as mean \pm SD. Significance was accepted at levels of $P<0.05$ and $P<0 \cdot 01$.

\section{RESULTS}

\section{In vivo susceptibility of aged mice to experimental $C$. albicans infection}

The effect of ageing on host resistance to systemic candidiasis was assessed by monitoring the survival curves of young and aged C57BL/6 mice intravenously infected with the high-virulence C. albicans strain ATCC 26555. Mice were challenged with $8 \times 10^{5}$ yeast cells per mouse and their survival was observed for 21 days (Fig. 1a). This dose was selected following preliminary studies evaluating the mortality rates in C57BL/6 young mice according to the number of C. albicans cells injected. Under these conditions, the percentage of dead animals was $83 \%$ in aged mice, whilst only $44 \%$ of the young mice had died at day 21 ; this statistically significant difference $(P=0.0031)$ indicated that old mice are more susceptible to invasive candidiasis in this experimental model. To investigate further the effect of ageing on disseminated candidiasis, mice were infected using the same conditions $\left(8 \times 10^{5}\right.$ yeast cells per mouse), killed at random on days 3 and 5 p.i. and the number of c.f.u. (g kidney) $)^{-1}$ was determined (Fig. 1b). The actual fungal growth in this target organ was significantly higher in aged mice compared with young mice on days 3 and 5, further supporting the high susceptibility of aged mice to invasive candidiasis. In spite of the higher fungal burden in the kidneys of aged infected mice, the level of TNF- $\alpha$ in the kidney homogenates was similar to that of young mice (Fig. 1c). Therefore, when TNF- $\alpha$ levels in kidney homogenates, expressed as levels of TNF- $\alpha\left(10^{6} \text { c.f.u. }\right)^{-1}$, were compared, a significant difference $(P<0 \cdot 05)$ was observed at day 3 between young $(3500 \pm 1100)$ and aged $(550 \pm 240)$ mice. Similar qualitative differences between young and aged mice concerning fungal burden and levels of TNF- $\alpha$ $\left(10^{6} \text { c.f.u. }\right)^{-1}$ in kidneys were obtained at day 3 following i.v. infection with a lower (sublethal) dose of C. albicans ATCC 26555 (not shown).

\section{Effect of ageing on in vitro TNF- $\alpha$ production by macrophages}

We have previously reported that Toll-like receptor 2 (TLR2) is the main receptor triggering in vitro TNF- $\alpha$ production by macrophages in response to C. albicans (Gil \& Gozalbo, 2006; Villamón et al., 2004b) and it has been (a)

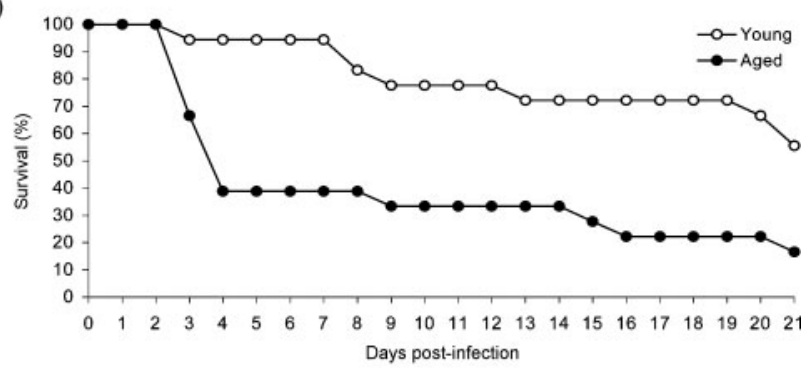

(b)

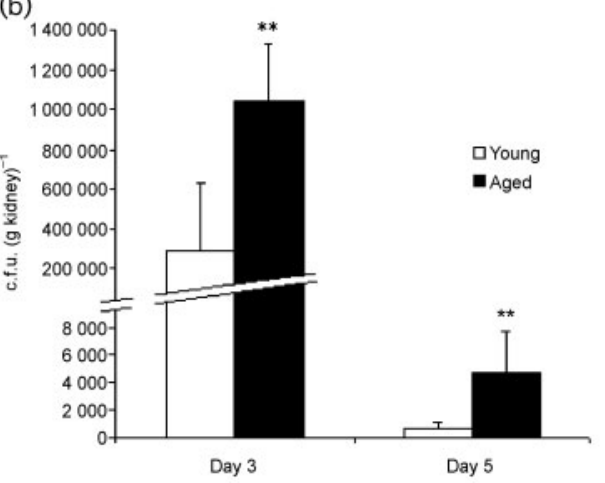

(c)

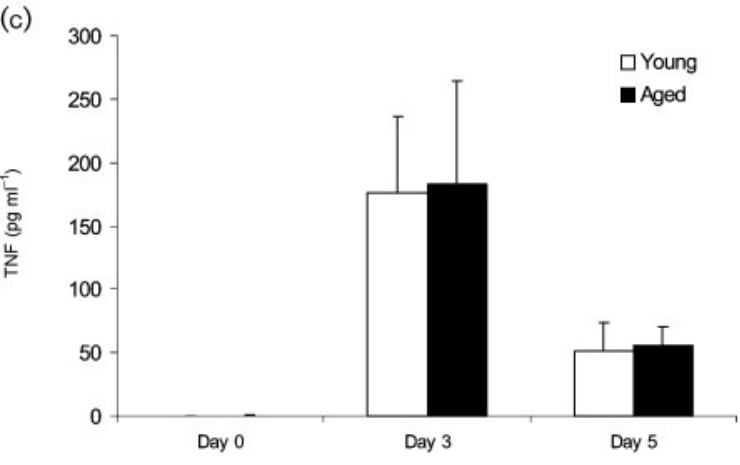

Fig. 1. In vivo susceptibility of aged mice to experimental $C$. albicans infection. (a) Young and aged animals were i.v. infected with $0.8 \times 10^{6}$ C. albicans ATCC 26555 yeast cells per mouse and survival was observed for 21 days. The data show one representative experiment of two, using 18 mice for each group. (b, c) Additional young and aged mice were identically infected and four mice from each group were killed on days 3 and 5 p.i. to assess the outgrowth of the yeast (b) and the levels of TNF- $\alpha$ (c) in the kidneys. TNF- $\alpha$ was also measured in the kidneys of two uninfected mice of each group (day $0)$. The results are shown as means \pm SD from one representative experiment of two. ${ }^{*}, P<0.01$.

described that ageing negatively skews macrophage TLR2and TLR4-mediated pro-inflammatory responses (Boehmer et al., 2004, 2005; Renshaw et al., 2002). Therefore, we checked the in vitro production of TNF- $\alpha$ by macrophages from young and aged mice in response to C. albicans (Fig. 2). Zymosan (a known TLR2 agonist) and LPS (a TLR4 agonist) were included as control stimuli. The synthesis of TNF- $\alpha$ in response to LPS and zymosan was significantly lower by macrophages from aged mice compared with the 


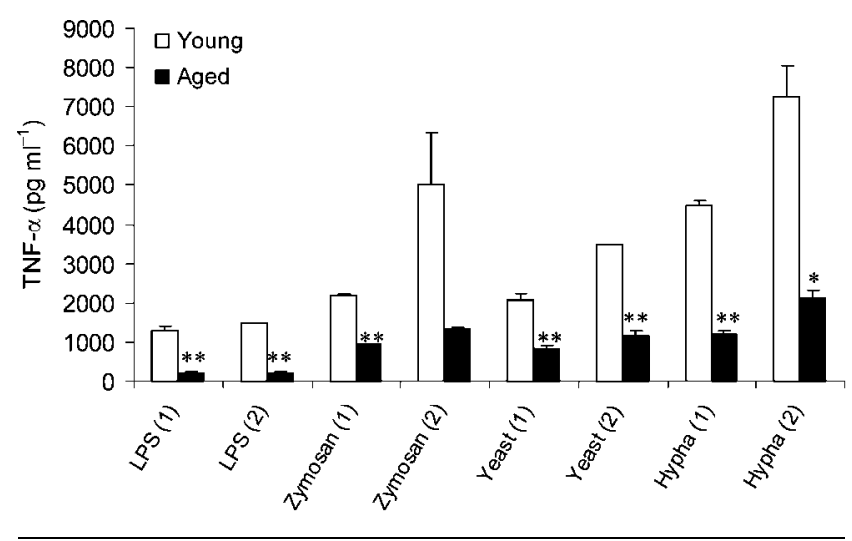

Fig. 2. TNF- $\alpha$ production by macrophages in response to $C$. albicans. Resident peritoneal macrophages from young and aged mice were challenged for $24 \mathrm{~h}$ with E. coli LPS (250 and $500 \mathrm{ng} \mathrm{ml}^{-1}$; samples 1 and 2 , respectively), zymosan $\left(3.75 \times 10^{6}\right.$ and $7.5 \times 10^{6}$ particles $\mathrm{ml}^{-1}$; samples 1 and 2 , respectively), heat-inactivated $C$. albicans yeast cells [150 and $300 \mu \mathrm{g}$ cells (dry wt) $\mathrm{ml}^{-1}$; samples 1 and 2, respectively] and heat-inactivated C. albicans hyphae [150 and $300 \mu \mathrm{g}$ cells (dry wt) $\mathrm{ml}^{-1}$; samples 1 and 2 , respectively]. The concentration of TNF- $\alpha$ in the culture supernatants was measured by ELISA. Results are shown as means $\pm S D$ of duplicates from one representative experiment of two. ${ }^{*}, P<0.05 ;{ }^{*}, P<0.01$.

synthesis by macrophages from young mice. Similarly, production of TNF- $\alpha$ by macrophages from aged mice in response to yeast cells and hyphae of $C$. albicans was also significantly impaired at all doses tested.

\section{Cytokine production by splenocytes from $\boldsymbol{C}$. albicans-infected aged mice}

To assess the production of cytokines upon primary infection in aged mice in comparison with young mice, animals were i.v. infected with the non-germinative, lowvirulence strain PCA2, and the in vitro levels of TNF- $\alpha$ and IFN- $\gamma$ production by $C$. albicans-stimulated splenocytes were assessed 3 days after infection. Splenocytes stimulated with LPS and zymosan served as controls. As described above for resident peritoneal macrophages, cells from aged mice showed severely impaired production of TNF- $\alpha$ in response to all stimuli tested: LPS (55\% inhibition), zymosan (70\% inhibition), yeast cells (50-90\% inhibition, depending on the dose) and hyphae (47-87\% inhibition, depending on the dose). Also, as early as 3 days p.i., IFN- $\gamma$ production was dramatically impaired in aged mice compared with young mice, in response to LPS, zymosan, yeast cells and hyphae (Fig. 3a).

This assay was also performed after primary infection of mice with a sublethal dose of the high-virulence $C$. albicans strain ATCC 26555. In vitro production of IFN- $\gamma$ by splenocytes was also greatly diminished in aged mice in response to all stimuli tested (Fig. 3b). As described previously, mice produced more IFN- $\gamma$ upon exposure to

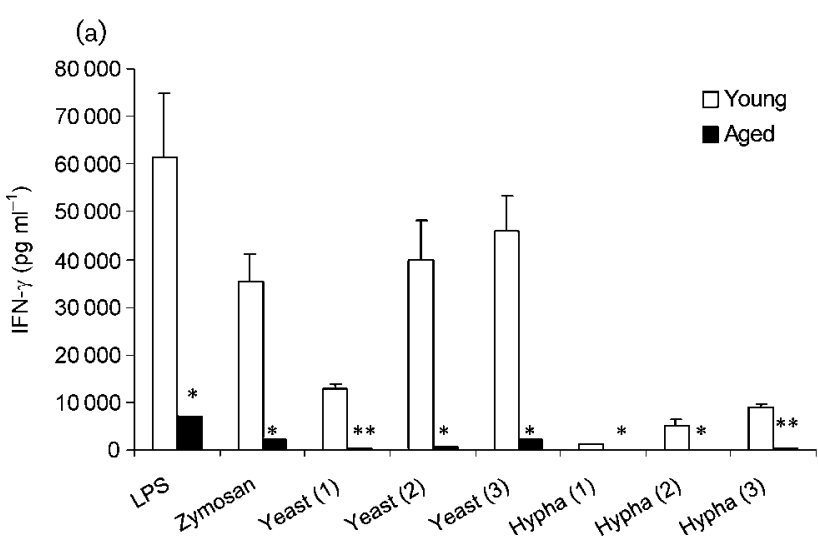

(b)

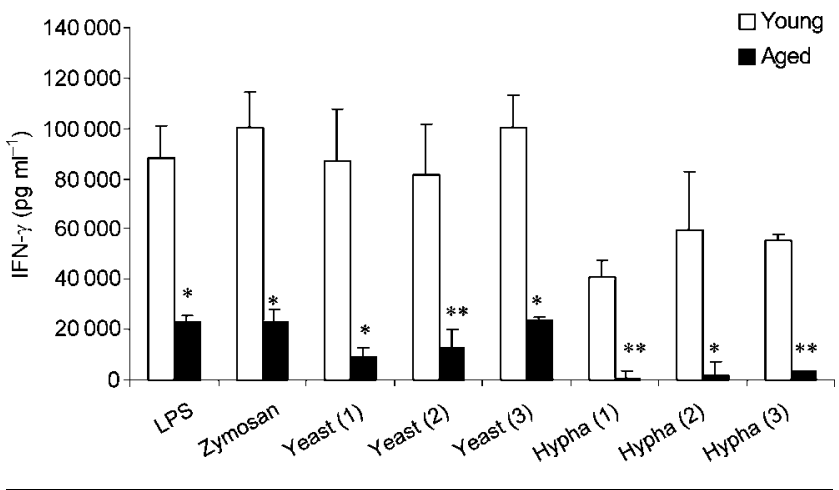

Fig. 3. IFN- $\gamma$ production by splenocytes of infected mice in response to $C$. albicans. Splenocytes from young and aged mice previously infected with strain PCA2 (a) or ATCC 26555 (b) were challenged for $48 \mathrm{~h}$ with LPS $\left(250 \mathrm{ng} \mathrm{ml}^{-1}\right)$, zymosan $\left(3.75 \times 10^{6}\right.$ particles $\left.\mathrm{ml}^{-1}\right)$, heat-inactivated C. albicans yeast cells $\left[15,30\right.$ or $60 \mu \mathrm{g}$ cells (dry wt) $\mathrm{ml}^{-1}$; samples 1,2 and 3 , respectively] or heat-inactivated $C$. albicans hyphae $[15,30$ or $60 \mu \mathrm{g}$ cells (dry wt) $\mathrm{ml}^{-1}$; samples 1,2 and 3 , respectively]. The concentration of IFN- $\gamma$ in the culture supernatants was measured by ELISA. Results are shown as means \pm SD of duplicates from one representative experiment of two. ${ }^{*}, P<0.05$; ${ }^{\star}, P<0.01$

yeast cells than upon exposure to hyphae (Fig. 3a, b) (Romani, 2004; Romani et al., 1991; Villamón et al., 2004a).

\section{Effect of ageing on development of the Th1 response in mice}

To define the effect of ageing on development of the Th1 anticandidal response, we determined the frequency of IFN$\gamma$-producing $\mathrm{CD}^{+}{ }^{+} \mathrm{T}$ lymphocytes in mice infected with $C$. albicans. To this purpose, mice were infected i.v. with the lowvirulence strain PCA2; 3 days after infection, total spleen cells were challenged in vitro with heat-killed C. albicans ATCC 26555 yeast cells or hyphae for $18 \mathrm{~h}$ and analysed for IFN- $\gamma$ secretion using the mouse IFN- $\gamma$-secretion flow cytometric assay. The cells were simultaneously labelled 
with FITC-conjugated $\mathrm{CD} 4 \mathrm{mAb}$ and $\mathrm{PE}-\mathrm{Cy} 5$-conjugated CD3 mAb. As shown in Fig. 4(a), the number of IFN- $\gamma$ producing $\mathrm{CD}^{+} \mathrm{T}$ lymphocytes in C. albicans-infected mice was significantly reduced in aged mice compared with young mice in response to both yeast cells and hyphae. A similar result was obtained when the frequency of IFN- $\gamma$ producing $\mathrm{CD} 4^{+} \mathrm{T}$ lymphocytes was determined following infection of mice with a sublethal dose of the high-virulence C. albicans strain ATCC 26555 (Fig. 4b). As expected, the frequency of IFN- $\gamma$-producing $\mathrm{CD}^{+}{ }^{+} \mathrm{T}$ lymphocytes was higher in response to yeast cells than in response to hyphae, both in aged and young mice infected with C. albicans PCA2 or ATCC 26555. These results were in accordance with the in vitro levels of IFN- $\gamma$ produced by splenocytes (see Fig. 3).

\section{Immunization with PCA2 strain induces anti-C. albicans resistance in aged mice}

In order to investigate the effect of ageing on the ability to develop an acquired resistance upon vaccination (primary sublethal infection), we used a well-characterized model in which substantial protection against reinfection with highly virulent wild-type yeast cells is induced by prior exposure of mice to a low-virulence agerminative strain of $C$. albicans (Romani et al., 1991; Villamón et al., 2004c). Aged mice were i.v. injected with $10^{6}$ cells of the low-virulence strain PCA2 and their survival was monitored for 15 days (not shown). All aged mice survived this infection and appeared to be clinically normal. Secondary infection was performed 15 days after the primary i.v. challenge by i.v. injection of $10^{6}$ cells of the high-virulence strain ATCC 26555 and survival was monitored for 14 days (Fig. 5). Naive aged mice i.v. injected with $10^{6}$ cells of the high-virulence strain ATCC 26555 (primary candidiasis in naive mice) were used as controls (Fig. 5). Aged mice surviving PCA2 infection (vaccinated) were resistant to C. albicans ATCC 26555 infection ( $90 \%$ survivors at day 14), whereas $100 \%$ of control aged mice primarily infected with the high-virulence strain (non-vaccinated) died within 8 days. As a control, an identical immunization protocol was performed in parallel assays with young mice and, as expected, $100 \%$ survival was observed at day 14 after secondary infection (not shown). These results suggest that although effector mechanisms of resistance to primary C. albicans infection are impaired in aged mice, they are still able to develop a significant acquired resistance upon vaccination (primary sublethal infection).
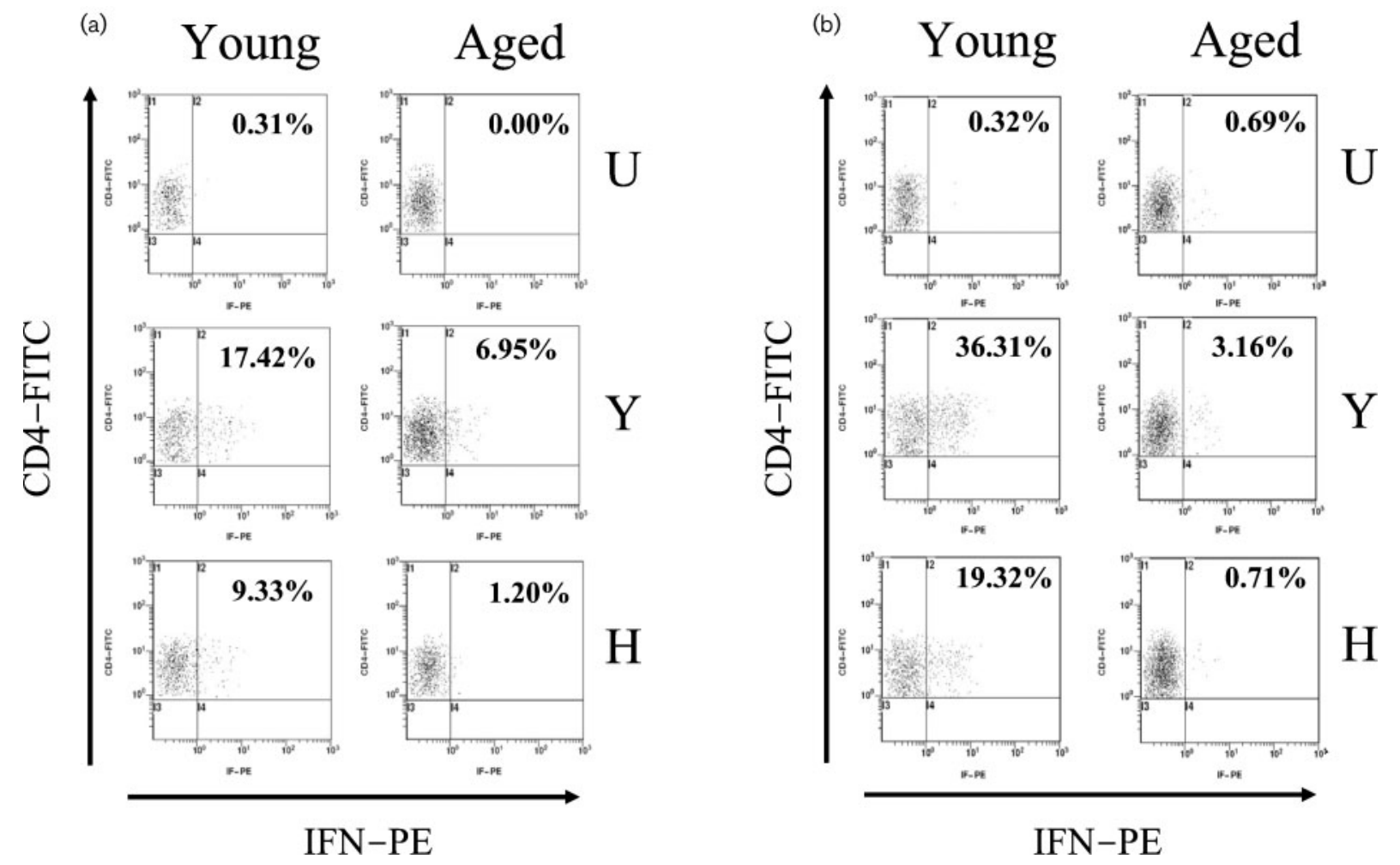

Fig. 4. Detection of IFN- $\gamma$-secreting $C D 4^{+} \mathrm{T}$ cells in response to C. albicans. Total spleen cells of PCA2-infected (a) or ATCC 26555-infected (b) young and aged mice were left unstimulated (U) or challenged in vitro with $30 \mu \mathrm{g}$ cells (dry wt) $\mathrm{ml}^{-1}$ of heat-inactivated C. albicans yeast cells $(\mathrm{Y})$ or hyphae $(\mathrm{H})$ for $18 \mathrm{~h}$. Secretion of IFN- $\gamma$ (IFN-PE) was examined using a mouse IFN- $\gamma$-secretion assay as described in Methods. IFN- $\gamma$ secretion was analysed on electronically gated CD ${ }^{+}$and $\mathrm{CD}^{+}{ }^{+}$cells by flow cytometry. Percentages reflect the number of IFN- $\gamma$-secreting $\mathrm{CD} 4^{+}$cells. The data shown are from one representative experiment of two. 


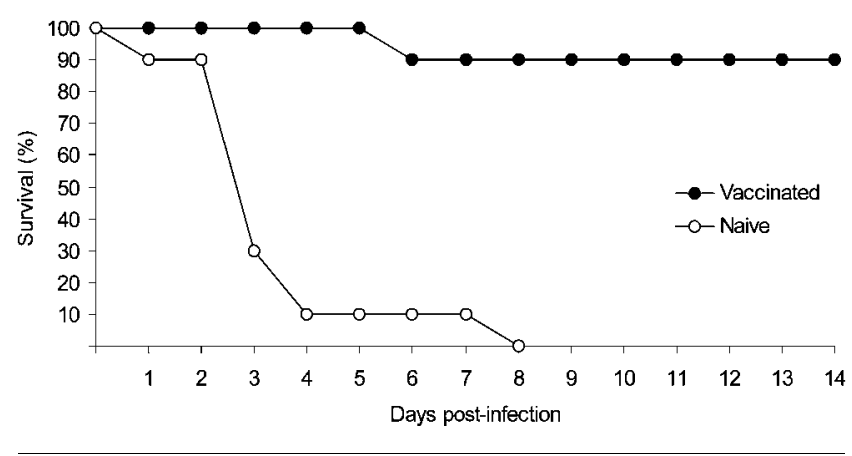

Fig. 5. Survival of vaccinated aged mice to secondary infection. Mice were vaccinated by i.v. infection with $10^{6}$ cells of the low-virulence $C$. albicans strain PCA2 15 days before reinfection. Naive or vaccinated aged mice were i.v. infected with $10^{6}$ C. albicans ATCC 26555 yeast cells per mouse and survival was observed for 14 days. The graph shows data from one representative experiment of two, using ten mice for each group.

\section{C. albicans-specific antibodies in aged immunized mice}

C. albicans-specific antibodies were measured in vaccinated mice to assess the ability of aged mice to mount a humoral response. Sera were collected from aged and young mice 14 days after the secondary infection and C. albicansspecific IgG was detected by Western immunoblotting using soluble cell extracts from yeast cells or hyphae (Fig. 6). Control sera from uninfected aged mice showed a higher background than control sera from uninfected young mice tested at the same dilution. The diversity of antigens, from both yeast cells and hyphae, recognized by sera from aged infected mice was clearly diminished when compared with that recognized by sera from young infected mice. The number of bands and the intensity of the staining were lower with sera from aged mice than with sera from young mice.

\section{DISCUSSION}

As deterioration of immune function and increased incidence and lethality of infectious diseases in the elderly is well documented (Effros, 2001; Ginaldi et al., 2001), in this report we examined the effect of ageing in a model of haematogenously disseminated candidiasis in C57BL/6 mice. Within the growing body of literature on changing immune function with ageing, this study is the first to provide a detailed analysis of invasive candidiasis in aged mice.

Our model of disseminated candidiasis demonstrated that ageing significantly increased the overall susceptibility of mice to systemic C. albicans infection. Decreased survival of aged mice compared with young mice was evident and a higher fungal burden in the kidneys of aged infected mice was detected. However, the level of TNF- $\alpha$ measured in homogenates from the kidneys of aged mice was similar to that of young mice, in spite of their higher fungal burden.
These results suggested a lower TNF- $\alpha$ production by cells from aged mice in response to $C$. albicans infection, as deduced from a comparison of the levels of TNF- $\alpha$ $\left(10^{6} \text { c.f.u. }\right)^{-1}$.

It has been described that aged macrophages secrete significantly lower levels of pro-inflammatory cytokines than young macrophages in response to different ligands of TLRs (Boehmer et al., 2004, 2005; Renshaw et al., 2002), although different explanations have been reported for this observation. Renshaw et al. (2002) found that macrophages from aged mice expressed lower levels of TLRs, whereas Boehmer et al. $(2004,2005)$ concluded that decreased expression of mitogen-activated protein kinases could be the mechanism responsible for age-related deterioration of TLR-mediated signalling. We have previously described that TLR2 is the main receptor triggering TNF- $\alpha$ in macrophages in response to C. albicans (Gil \& Gozalbo, 2006; Villamón et al., 2004b) and that pro-inflammatory cytokines, such as TNF- $\alpha$, secreted by activated macrophages are critical in protecting the host against disseminated candidiasis (Netea et al., 1999; Steinshamn et al., 1996). Therefore, we determined the effect of ageing on signalling cellular pathways for TNF- $\alpha$ production upon $C$. albicans recognition by macrophages. Our results clearly showed a reduction in TNF- $\alpha$ production in vitro by resident peritoneal macrophages from aged mice in response to both yeast cells and hyphae of $C$. albicans, supporting decreased production of TNF- $\alpha$ in vivo during primary infection, as mentioned above. Therefore, this impaired TNF- $\alpha$ production may be partly responsible for the increased susceptibility of aged mice to candidiasis.

In addition, levels of TNF- $\alpha$ and IFN- $\gamma$ production by $C$. albicans-stimulated splenocytes after infection with strain PCA2 were dramatically impaired in aged mice compared with young mice. This result indicated that, early in infection, production of pro-inflammatory cytokines, including the Th1 cytokine IFN- $\gamma$, is impaired in aged mice. To correlate these findings with the acquired immunity to $C$. albicans, we measured the frequency of IFN- $\gamma$-producing CD4 ${ }^{+}$T lymphocytes in mice i.v. infected with the low-virulence strain PCA2, which induces a Th1acquired immune response (Romani et al., 1991). Our results showed a greatly diminished frequency of IFN- $\gamma$ producing $\mathrm{CD} 4{ }^{+} \mathrm{T}$ cells in aged mice compared with young mice, indicating that aged mice developed an impaired protective Th1 cellular response against $C$. albicans infection. In order to determine whether this impaired Th1 response may help explain why aged mice were more susceptible to primary infection with a high-virulence strain, we investigated the development of a Th1 response following infection with a sublethal dose of the virulent $C$. albicans strain ATCC 26555. In this case, aged mice also showed an impaired specific Th1 response and lower levels of IFN- $\gamma$, indicating that this defect may be involved directly in the susceptibility of aged mice to primary candidal infection. 


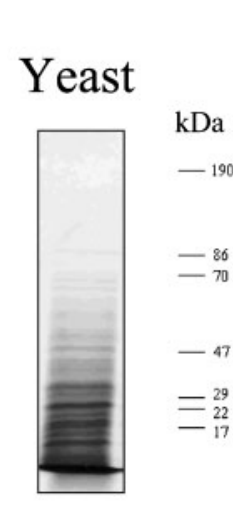

\section{Hyphae}

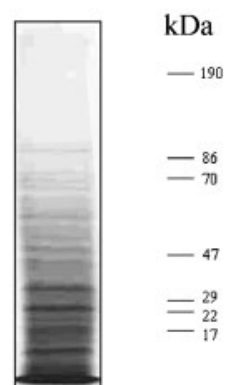

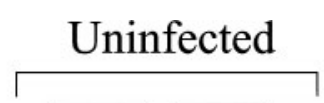

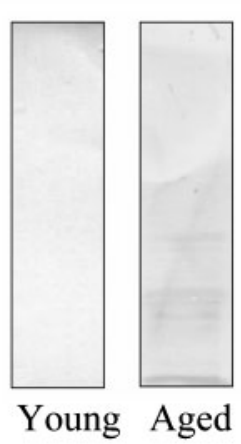

Uninfected

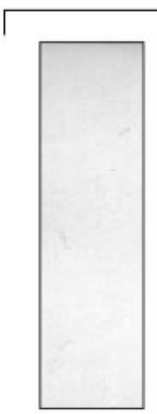

Young

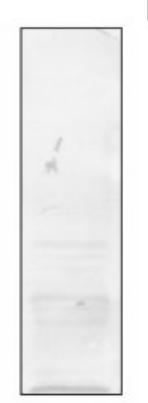

Aged
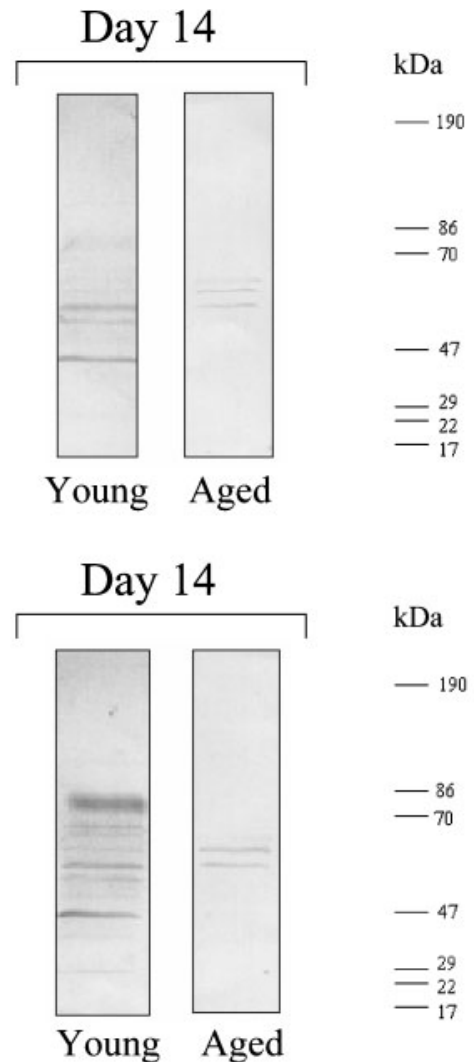

Fig. 6. Effect of ageing on the humoral immune response in C. albicans-vaccinated mice. C. albicans-specific IgG in sera was assayed by Western blotting of soluble cell extracts obtained from yeast cells or hyphae. Each serum sample was a pool from four randomly selected uninfected or infected mice (at day 14 after secondary infection). C. albicans extracts stained with Coomassie brilliant blue to visualize proteins are shown. The positions of molecular mass markers are indicated.

We also studied the effect of ageing in the development of the acquired humoral response against $C$. albicans during secondary invasive infection. Sera from uninfected aged mice showed a higher background than sera from uninfected young mice, probably as a consequence of the increase in the levels of serum immunoglobulins that has been described in elderly subjects (Ginaldi et al., 2001). At day 15 after secondary infection, IgG titres were similar in sera from both aged and young mice (data not shown); however, the pattern of C. albicans antigens recognized by Western blot analysis was different. Sera from infected aged mice recognized fewer antigenic proteins than sera from infected young mice, in both yeast and hyphal extracts. This result may be explained by a decreased helper T-cell function and also by an intrinsic primary B-cell deficit (the ageingassociated decrease in the number of total B lymphocytes and in the high-affinity protective antibody response) (Ginaldi et al., 2001; McGlauchlen \& Vogel, 2003). Therefore, the acquired humoral response to C. albicans was modified in aged mice compared with young mice; however, there was no evidence indicating whether this might account for the high susceptibility of aged mice to primary candidiasis, as antibody responses to C. albicans are complex, with the presence in immune sera of protective, non-protective and deleterious antibodies (Bromuro et al., 2002). Although it is accepted that the humoral response is not critical for host defence against $C$. albicans during primary systemic infection, administration of antibodies against certain C. albicans antigens confers protection in naive mice against infection, and also other C. albicans epitopes have been used for antibody-mediated protection against systemic candidiasis (Bromuro et al., 2002; Matthews et al., 2003; Montagnoli et al., 2004; Torosantucci et al., 2005; Vilanova et al., 2004; Zhang et al., 2006). In spite of the impaired acquired Th1 immune response and modified humoral response in aged mice, they were still capable of mounting an acquired resistance upon vaccination by the primary sublethal model of infection with a low-virulence strain, indicating that, although ageing is associated with a decline in immune function, a protective response may still be conserved. We have previously described a similar result using $T L R 2^{-/-}$mice, which showed a diminished Th1 response, although it was still sufficient to confer acquired protection against secondary infection (Villamón et al., 2004c). As mentioned above, the role of antibodies in the development of a protective response upon vaccination is complex and therefore the role of altered antibody production by aged mice in protection remains to be established. 
In summary, we found that aged mice were more susceptible to in vivo experimental primary infection by $C$. albicans. This increased susceptibility was associated with defective synthesis of TNF- $\alpha$, a lower specific protective Th1 response, which resulted in lower levels of IFN- $\gamma$, and altered development of specific antibodies. However, aged mice were still capable of mounting an acquired resistance upon vaccination. Our results suggest that the ageing-associated decreased immune function may result in an increased susceptibility to fungal infections. Identification of the multiple molecular mechanisms underlying the age-associated decline of the immune function is required for the development of interventions designed to prevent or reverse immunosenescence, in order to decrease the incidence of these infections in elderly people.

\section{ACKNOWLEDGEMENTS}

This work was supported by grant PI04/1472 (Instituto de Salud Carlos III; Ministerio de Sanidad y Consumo, Spain). C. M. is the recipient of a fellowship from Ministerio de Educación y Ciencia, Spain.

\section{REFERENCES}

Boehmer, E. D., Goral, J., Faunce, D. E. \& Kovacs, E. J. (2004). Agedependent decrease in Toll-like receptor 4-mediated proinflammatory cytokine production and mitogen-activated protein kinase expression. J Leukoc Biol 75, 342-349.

Boehmer, E. D., Meehan, M. J., Cutro, B. T. \& Kovacs, E. J. (2005). Aging negatively skews macrophage TLR2- and TLR4-mediated proinflammatory responses without affecting the IL-2-stimulated pathway. Mech Ageing Dev 126, 1305-1313.

Bromuro, C., Torosantucci, A., Chiani, P., Conti, S., Polonelli, L. \& Cassone, A. (2002). Interplay between protective and inhibitory antibodies dictates the outcome of experimentally disseminated candidiasis in recipients of Candida albicans vaccine. Infect Immun 70, 5462-5470.

Calderone, R. A. (2001). Candida and Candidiasis. New York: American Society for Microbiology.

Effros, R. B. (2001). Ageing and the immune system. Novartis Found Symp 235, 130-139.

Garber, G. (2001). An overview of fungal infections. Drugs 61, 1-12. Gil, M. L. \& Gozalbo, D. (2006). TLR2, but not TLR4, triggers cytokine production by murine cells in response to Candida albicans yeasts and hyphae. Microbes Infect 8, 2299-2304.

Gil-Navarro, I., Gil, M. L., Casanova, M., O’Connor, J. E., Martínez, J. P. \& Gozalbo, D. (1997). The glycolytic enzyme glyceraldehyde-3phosphate dehydrogenase of Candida albicans is a surface antigen. J Bacteriol 179, 4992-4999.

Ginaldi, L., Loreto, M. F., Corsi, M. P., Modesti, M. \& De Martinis, M. (2001). Immunosenescence and infectious diseases. Microbes Infect 3, 851-857.

Gozalbo, D., Gil-Navarro, I., Azorín, I., Renau-Piqueras, J., Martínez, J. P. \& Gil, M. L. (1998). The cell wall-associated glyceraldehyde-3phosphate dehydrogenase of Candida albicans is also a fibronectin and laminin binding protein. Infect Immun 66, 2052-2059.
Kauffman, C. A. (2001). Fungal infections in older adults. Clin Infect Dis 33, 550-555.

Matthews, R. C., Rigg, G., Hodgetts, S., Carter, T., Chapman, C., Gregory, C., Illidge, C. \& Burnie, J. (2003). Preclinical assessment of the efficacy of Mycograb, a human recombinant antibody against fungal HSP90. Antimicrobial Agents Chemother 47, 2208-2216.

McGlauchlen, K. S. \& Vogel, L. A. (2003). Ineffective humoral immunity in the elderly. Microbes Infect 5, 1279-1284.

Montagnoli, C., Sandini, S., Bacci, A., Romani, L. \& La Valle, R. (2004). Immunogenicity and protective effect of recombinant enolase of Candida albicans in a murine model of systemic candidiasis. Med Mycol 42, 319-324.

Netea, M. G., van Tits, L. J. H., Curfs, J. H. A. J., Amiot, F., Meis, J. F. G. M., van der Meer, J. W. M. \& Kullberg, B. J. (1999). Increased susceptibility of TNF- $\alpha$ lymphotoxin- $\alpha$ double knockout mice to systemic candidiasis through impaired recruitment of neutrophils and phagocytosis of Candida albicans. J Immunol 163, 1498-1505.

Nucci, M., Colombo, A. L., Silveira, F., Richtmann, R., Salomão, R., Branchini, M. L. \& Spector, N. (1998). Risk factors for death in patients with candidemia. Infect Control Hosp Epidemiol 11, 846-850.

Plackett, T. P., Boehmer, E. D., Faunce, D. E. \& Kovacs, E. J. (2004). Aging and innate immune cells. J Leukoc Biol 76, 291-299.

Renshaw, M., Rockwell, J., Engleman, C., Gewirtz, A., Katz, J. \& Sambhara, S. (2002). Cutting edge: impaired Toll-like receptor expression and function in aging. J Immunol 169, 4697-4701.

Romani, L. (2004). Immunity to fungal infections. Nat Rev Immunol 4, 1-23.

Romani, L., Mocci, S., Bietta, C., Lanfaloni, L., Puccetti, P. \& Bistoni, F. (1991). Th1 and Th2 cytokine secretion patterns in murine candidiasis: association of Th1 responses with acquired resistance. Infect Immun 59, 4647-4654.

Steinshamn, S., Bemelmans, M. H. A., Van Tits, L. H. J., Bergh, K., Buurman, W. A. \& Waage, A. (1996). TNF receptors in murine Candida albicans infection: evidence for an important role of TNF receptor p55 in antifungal defense. J Immunol 157, 2155-2159.

Torosantucci, A., Bromuro, C., Chiani, P. \& 9 other authors (2005). A novel glyco-conjugate vaccine against fungi pathogens. $J$ Exp Med 202, 597-606.

Vilanova, M., Teixeira, L., Caramalho, I. \& 7 other authors (2004). Protection against systemic candidiasis in mice immunized with secreted aspartic proteinase 2. Immunology 111, 334-342.

Villamón, E., Gozalbo, D., Roig, P., Murciano, C., O’Connor, J. E., Fradelizi, D. \& Gil, M. L. (2004a). Myeloid differentiation factor 88 (MyD88) is required for murine resistance to Candida albicans and is critically involved in Candida-induced production of cytokines. Eur Cytokine Netw 15, 263-271.

Villamón, E., Gozalbo, D., Roig, P., O’Connor, J. E., Fradelizi, D. \& Gil, M. L. (2004b). Toll-like receptor-2 is essential in murine defenses against Candida albicans infections. Microbes Infect 6, 1-7.

Villamón, E., Gozalbo, D., Roig, P., O’Connor, J. E., Ferrandiz, M. L., Fradelizi, D. \& Gil, M. L. (2004c). Toll-like receptor 2 is dispensable for acquired host immune resistance to Candida albicans in a murine model of disseminated candidiasis. Microbes Infect 6, 542-548.

Zhang, M. X., Bohlman, M. C., Itatani, C., Burton, D. R., Parren, P. W. H. I., St Jeor, S. C. \& Kozel, T. R. (2006). Human recombinant antimannan immunoglobulin G1 antibody confers resistance to hematogenously disseminated candidiasis in mice. Infect Immun 74, $362-369$. 